\title{
Release of PLGA-encapsulated dexamethasone from microsphere loaded porous surfaces
}

\author{
G. J. S. Dawes · L. E. Fratila-Apachitei • \\ B. S. Necula · I. Apachitei - G. J. Witkamp • \\ J. Duszczyk
}

Received: 16 February 2009/Accepted: 27 July 2009/Published online: 11 August 2009

(c) The Author(s) 2009. This article is published with open access at Springerlink.com

\begin{abstract}
The aim of the present study was to investigate the morphology and function of a drug eluting metallic porous surface produced by the immobilization of poly lactide-co-glycolide microspheres bearing dexamethasone onto plasma electrolytically oxidized $\mathrm{Ti}-6 \mathrm{Al}-7 \mathrm{Nb}$ medical alloy. Spheres of $20 \mu \mathrm{m}$ diameter were produced by an oilin-water emulsion/solvent evaporation method and thermally immobilized onto titanium discs. The scanning electron microscopy investigations revealed that the size distribution and morphology of the attached spheres had not changed significantly. The drug release profiles following degradation in phosphate buffered saline for $1000 \mathrm{~h}$ showed that, upon immobilisation, the spheres maintained a sustained release, with a triphasic profile similar to the non-attached system. The only significant change was an increased release rate during the first $100 \mathrm{~h}$. This difference was attributed to the effect of thermal attachment of the spheres to the surface.
\end{abstract}

\section{Introduction}

During 2007, there were more than 150,000 patients requiring total or partial joint arthroplasty in the United

G. J. S. Dawes $(\bowtie) \cdot$ L. E. Fratila-Apachitei ·

B. S. Necula · I. Apachitei · J. Duszczyk

Department of Materials Science and Engineering,

Delft University of Technology, Mekelweg 2, 2628 CD Delft,

The Netherlands

e-mail: G.J.S.Dawes@tudelft.nl

G. J. Witkamp

Laboratory for Process Equipment, Delft University

of Technology, Leeghwaterstraat 44, 2628 CA Delft,

The Netherlands
Kingdom alone [1]. At the same time, 13,000 patients required revision surgery, many of these due to septic or aseptic implant loosening. One way to reduce this proportion is to improve implant fixation. Previous work has been undertaken to control cellular activity by the generation of micro- or nanotexture onto the surface of metallic implants [2]. There is hope that drug eluting medical combination devices may afford even greater fixation by means of triggering specific cellular interactions next to providing additional biofunctionalities (e.g. antibacterial activity).

Poly (lactide-co-glycolide) (PLGA) has been studied since the 1980s [3] as a carrier for sustained drug release. It is known to degrade in water over time via hydrolytic fission [4], and the end products of this reaction are lactic and glycolic acid. Both of these chemicals are readily soluble in water and are excreted by mammals. These properties make PLGA a very attractive implant material, as no surgery is needed to remove the implant after its function is no longer required.

PLGA is known to show good chemical biocompatibility in animal studies due to a limited inflammatory response [5, 6]. As a result, PLGA has been used for many years as an implantable material, capable of being degraded in situ and require no further intervention for removal. A common example of this is its use as restorable sutures [7]. The degradation is controlled by local $\mathrm{pH}$ and polymer hydrophilicity. Some papers report an unusually large amount of inflammation from PLGA implants [8], usually attributed to the falling $\mathrm{pH}$ during polymer degradation. In an attempt to reduce this effect, research has been performed on modification of the PLGA matrix by the incorporation of basic salts $[9,10]$.

As a semicrystalline polymer, PLGA can also incorporate small hydrophobic molecules into its structure and 
release them over time when exposed to an aqueous solution by a combination of diffusion and degradation [11]. It can also be processed to encapsulate small aqueous droplets of hydrophilic drugs [12], or as a suspension of dry drug particles $[13,14]$. The last two techniques are suitable for delivering proteins, whilst all three are suitable for delivery of lower molecular weight drugs. Whilst microspheres are usually used to deliver drugs to a single site of action, nanospheres have been used for drug delivery in cases needing considerable diffusion, such as intraocularly [15] or as an aerosol [16].

PLGA can be used to deliver drugs in the form of films or microparticles. PLGA films can be used on metallic surfaces to some effect, particularly in stents where sustained delivery of drugs is critical in preventing restenosis [17]. However, this type of delivery method results in an uncontrolled, usually short, diffusion-like release and can disguise the structure of the metallic implant by applying a uniform layer to the surface [18]. Whilst this may help to prevent cell proliferation in stents, in orthopedic applications it has been shown that a micro/nanostructure is very important for bone growth, even on the surface of a PLGA film [2]. An alternate method of sustained drug delivery would be to employ a particulate formulation of PLGA. By doing so, it would allow for a controlled, tunable drug release whilst preserving or enhancing the underlying texture of the implant surface, thus also promoting cellular interactions.

Titanium and its medical alloys have been the choice for many years for implanted devices as it displays very good biocompatibility relative to other metallic biomaterials such as surgical steel and cobalt-chrome alloys. They are used in a range of fields such as dental prostheses, total joint replacements as well as bone fixation devices [19]. Titanium surfaces can be modified by various processes (e.g. plasma spray deposition, sintering, plasma electrolytic oxidation) to increase the roughness of the titanium surface or create porous topographies allowing for bone on/in growth or enhanced cellular interaction [20].

The aim of the work in this paper is to investigate the morphology and function of a drug eluting metallic porous surface, produced by the immobilization of PLGA microspheres bearing a drug onto plasma electrolytically oxidized titanium. Research has been performed on these types of surfaces before, albeit using a film or surfacecovering layer to do so [21, 22]. The advantage to using a particulate drug delivery design would be to allow the incorporation of sustained drug delivery whilst preserving the controlled porous topography. The electrolyte chosen for plasma electrolytic oxidation was calcium acetate and calcium glycerophosphate, known to introduce calcium and phosphates in the oxide layers that can act as precursors for hydroxyapatite formation [23]. The PLGA microspheres were loaded with dexamethasone, a corticosteroid previously indentified with the differentiation of osteoblasts [24], as a restenosis inhibitor [25] and with the reduction of inflammatory responses [26]. Previous work performed on these spheres in an unattached, free-in-solution system [27] has shown that they can act as a sustained dexamethasone releasing system.

\section{Materials and methods}

\subsection{Materials}

Poly (lactide-co-glycolide) (MW: 40,000-75,000, 50:50), dexamethasone (98\%), poly (vinyl alcohol) (MW: 30,00070,000), sodium azide, disodium hydrogen phosphate, sodium dihydrogen phosphate, acetic acid, sodium acetate and all solvents were purchased from Sigma Aldrich chemicals. Distilled, deionised water $\left({ }_{d d} \mathrm{H}_{2} \mathrm{O}\right)$ was provided from Millipore.

Phosphate buffered saline (PBS) was made up with an additional $0.1 \mathrm{~g} / 1$ sodium azide.

\subsection{Microspheres synthesis}

Following on from our previous work [27], the synthesis of the spheres can be summarised as follows:

$400 \mathrm{mg}$ PLGA and $80 \mathrm{mg}$ dexamethasone were co-dissolved in $40 \mathrm{ml}$ dichloromethane:methanol 9:1. This solution was added in $5 \mathrm{ml}$ fractions to $100 \mathrm{ml} 0.2 \% \mathrm{w} / \mathrm{v}$ PVA solution in ${ }_{d d} \mathrm{H}_{2} \mathrm{O}$, and stirred at $1250 \mathrm{rpm}$ for $30 \mathrm{mins}$, followed by stirring at $60 \mathrm{rpm}$ for $18 \mathrm{~h}$. The microspheres were then filtered out of solution, washed with ${ }_{d d} \mathrm{H}_{2} \mathrm{O}$, and dried fully in a lyophiliser for $24 \mathrm{~h}$.

\subsection{Titanium plasma electrolytic oxidation and sphere immobilisation}

Ti-6Al-7Nb medical alloy (ACNIS International, France) was used as the substrate in the form of cylindrical disks with a thickness of $8 \mathrm{~mm}$ and a diameter of $19 \mathrm{~mm}$. The disks were ground with 1,200 grit paper (Struers, Denmark) using water as lubricating liquid. The samples were ultrasonically cleaned, washed in ethanol and deionised water, then dried under compressed air.

The plasma electrolytic oxidation (PEO) process was carried out in a double-wall glass electrolytic cell with an internal volume of $800 \mathrm{ml}$. The electrolyte used was a solution of $4.2 \mathrm{~g} / \mathrm{l}$ calcium glycerophosphate and $24 \mathrm{~g} / \mathrm{l}$ calcium acetate in deionised water (Sigma Aldrich).

Titanium disks were suspended in the centre of the electrolytic cell by screwing them to an insulated metallic rod acting as an anode whilst a cylindrical steel jacket in 
the solution acted as a cathode. The temperature of the electrolyte was maintained in the range of $15-25^{\circ} \mathrm{C}$ during oxidation by water circulation through the cell jacket, whilst the electrolyte was stirred at a speed of $500 \mathrm{rpm}$ to maintain homogeneity.

Oxidation was performed under galvanostatic conditions using a current density of $20 \mathrm{~A} / \mathrm{dm}^{2}$ for $5 \mathrm{~min}$, using an AC $(50 \mathrm{~Hz})$ power supply type ACS 1500 (ET Power Systems Ltd, UK). After oxidation, the samples were thoroughly cleaned with deionised water and dried in air.

Suspensions of the spheres were made up in ${ }_{\mathrm{dd}} \mathrm{H}_{2} \mathrm{O}$ with 1 min of sonication at $20 \mathrm{~W}$. Samples of $0.8 \mathrm{ml}$ were taken from this suspension and dropped onto one face of the titanium discs as prepared above to form one continuous droplet over the entire surface. This was repeated for each oxidised and non-oxidised sample. These samples were placed into an oven (Nabertherm) to allow the water to evaporate and for the microspheres to bond with the surface oxide. The thermal treatment applied was optimised around the $\mathrm{T}_{\mathrm{g}}\left(45^{\circ} \mathrm{C}\right)$ of the PLGA used $(1 \mathrm{~h}$ at $\left.50^{\circ} \mathrm{C}\right)$.

\subsection{Scanning electron microscopy (SEM) and size analysis}

Pictures of the microspheres loaded onto the titanium samples were obtained using a JEOL 6500 SEM at 5-15 kV. Each sample was gold sputter coated before analysis, and pictures were taken at 750-1000 $\times$ magnification. The images were analysed by Analysis software in order to produce a size distribution of the bonded spheres for each sample. At least 150 data points were used to get an accurate distribution of sizes.

\subsection{High performance liquid chromatography (HPLC)}

Samples of PBS or acetonitrile (for calibration) had their dexamethasone concentration analysed by HPLC, and thus were run on a machine fitted with a reverse phase Varian Chromosphere C18 column $(250 \times 4.6 \mathrm{~mm})$ with a running buffer of $60 \%$ acetonitrile, $40 \%$ sodium acetate buffer at $\mathrm{pH}$ 4.8. The concentration of dexamethasone was resolved using a Varian Prostar spectrometer reading at $254 \mathrm{~nm}$, with a dexamethasone peak at $3.75 \mathrm{~min}$. The machine was calibrated every day of use with at least three fresh samples of dexamethasone in acetonitrile of known concentrations straddling the expected concentrations.

\subsection{Surface loading efficiency}

Surface loading efficiency (SLE) was calculated using the following formula:
$S L E=\frac{d r u g \text { concentration on surface }}{\text { maximum expected drug concentration }} \times 100 \%$

A microsphere loaded titanium disc was washed with approx. $9 \mathrm{~g}$ acetonitrile and left to soak in the solvent for $24 \mathrm{~h}$ to ensure complete dissolution of all bonded microspheres. A $1.8 \mathrm{ml}$ sample was analysed by HPLC as noted in Sect. 2.5 for dexamethasone. Based on this result the total amount of attached spheres was estimated.

\subsection{Release profile assay}

Three samples of attached spheres on both oxidised and non-oxidised titanium discs were incubated in $10 \mathrm{ml}$ PBS at $\mathrm{pH} 7.0,37^{\circ} \mathrm{C}$ with $30 \mathrm{rpm}$ of stirring for $1000 \mathrm{~h}$. Samples of $1.7 \mathrm{ml}$ were removed and filtered through a $220 \mathrm{~nm}$ polyester membrane to remove any possible detached PLGA spheres. The incubated sample was then topped up to $10 \mathrm{ml}$ with fresh, filtered PBS.

Each removed sample was run on an HPLC as described in Sect. 2.5 with a dexamethasone peak at $3.75 \mathrm{~min}$, and a peak corresponding to the simulated body fluid at $1.76 \mathrm{~min}$.

\section{Results}

3.1 Morphology of the immobilised spheres

As found in our previous work [27], the applied microsphere synthesis scheme led to spheres produced of approximately $20 \mu \mathrm{m}$ diameter. Typical SEM images of the spheres attached to oxidised and non-oxidised titanium are shown in Fig. 1. A slight union between nearby spheres or partial melting of the spheres (Fig. 1a), showed the effect of thermal treatment onto the surface. This evidence suggests that the spheres are lightly attached to the titanium oxide surface texture. Areas with agglomerated spheres were occasionally observed. The distribution of sphere sizes after thermal attachment to the titanium oxide surface can be seen in Fig. 2. Before thermal treatment, the average diameter was $20 \pm 10 \mu \mathrm{m}$ [27], whilst after thermal treatment the sphere diameters were found to be $26 \pm 10 \mu \mathrm{m}$. These overlapping figures indicate that no significant change in diameter or shape of the spheres occurred due to thermal treatment.

\subsection{Surface loading}

The titanium oxide samples prepared in this work were loaded with approx. $10 \mathrm{mg}$ of the 20 spheres in order to obtain a monolayer of spheres on each surface. Two sphere-loaded titanium oxide samples were immersed in 

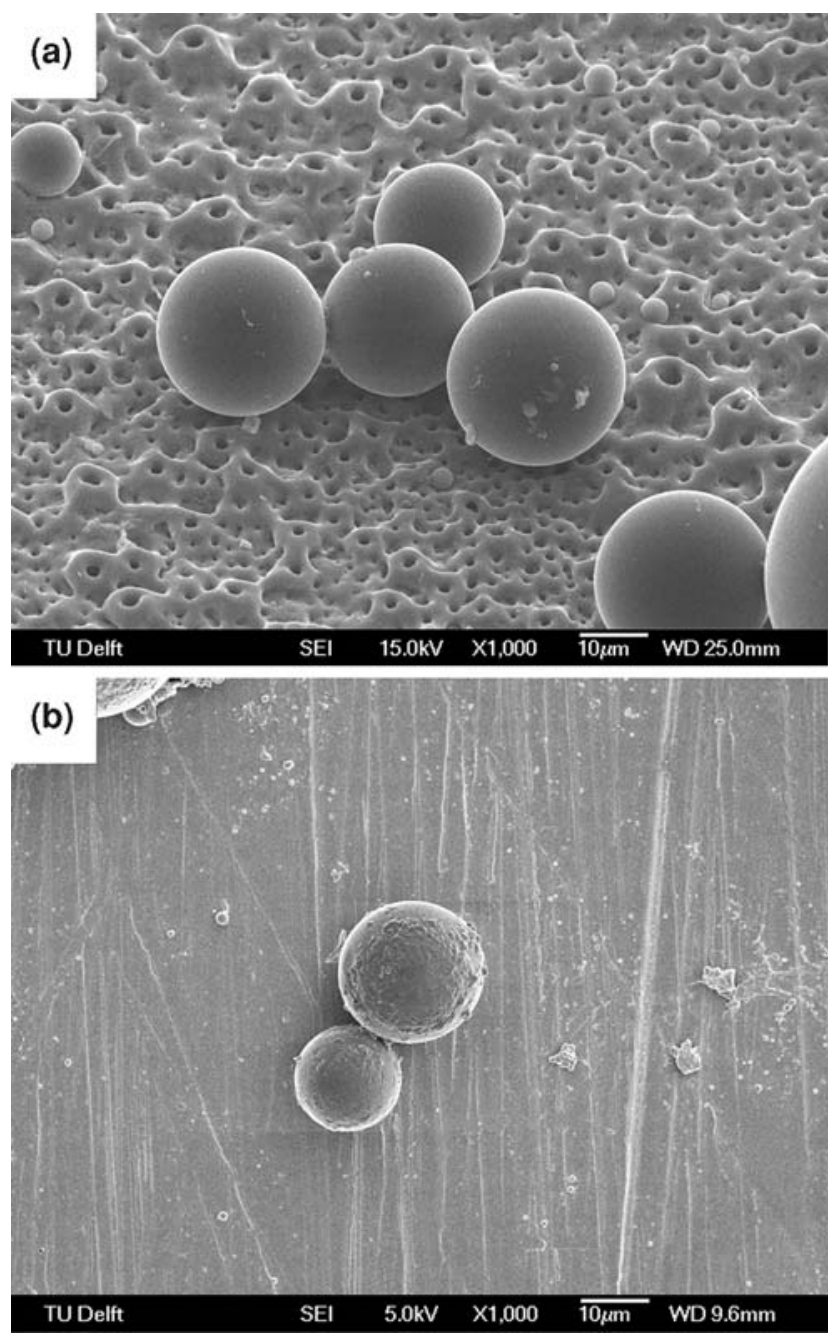

Fig. 1 Morphology of the immobilized PLGA spheres visualized by scanning electron microscopy: a $20 \mu \mathrm{m}$ spheres on an oxidised titanium surface $(15 \mathrm{kV}, \times 1000)$; b $20 \mu \mathrm{m}$ spheres on a non-oxidised titanium surface $(5 \mathrm{kV}, \times 1000)$

acetonitrile in order to assess the resulting dexamethasone concentration on the surface after thermal treatment, and thus by using the already known drug loading efficiency (1\% [27]), the amount of attached spheres. It was found that over $80 \%$ of the spheres remained attached to the surface after thermal treatment. Therefore, each $19 \mathrm{~mm}$ diameter disc was loaded with approximately $20 \mu \mathrm{g}$ of dexamethasone.

\subsection{In vitro drug release profile}

Figure 3 shows the release profile of dexamethasone from the immobilised PLGA spheres up to $1000 \mathrm{~h}$. It can be seen that this trend follows the general shape of previous work on non-attached systems $[11,27]$. However, a difference was observed between the free and immobilised $20 \mu \mathrm{m}$ spheres. At the very first hour, a dexamethasone release of

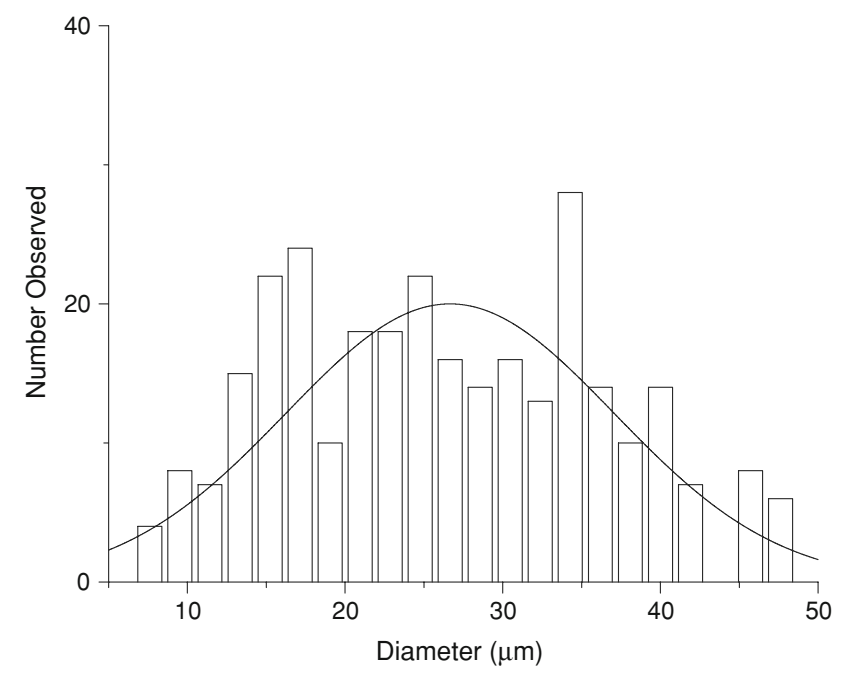

Fig. 2 Size distribution of the immobilised PLGA spheres. The values were calculated by visual analysis of the SEM images and include more than 150 data points

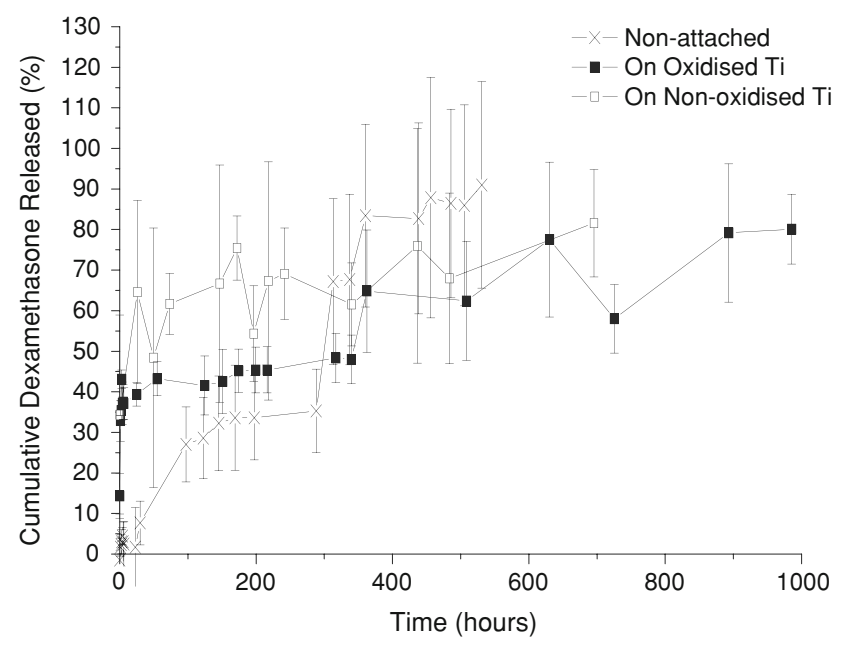

Fig. 3 Dexamethasone release profiles from the immobilised $20 \mu \mathrm{m}$ spheres on non-oxidised and oxidised titanium discs, plus nonimmobilised PLGA spheres (PBS, $37^{\circ} \mathrm{C}$ ). Average values with standard deviations are presented $(n=3)$

over $30 \%$ was reached for the attached spheres, whereas with the non-attached spheres almost no dexamethasone was released. Additionally, the release rate of both the attached sphere samples was compared to the non-attached system after $300 \mathrm{~h}(80 \%$ in $950 \mathrm{~h}$ vs. $90 \%$ in $550 \mathrm{~h})$. No significant differences have been identified between the release profiles from the oxidised and non-oxidised loaded discs.

\subsection{Sphere degradation}

Figure 4 shows typical SEM images of the immobilised spheres on porous oxidised titanium after $800 \mathrm{~h}$ of in vitro 


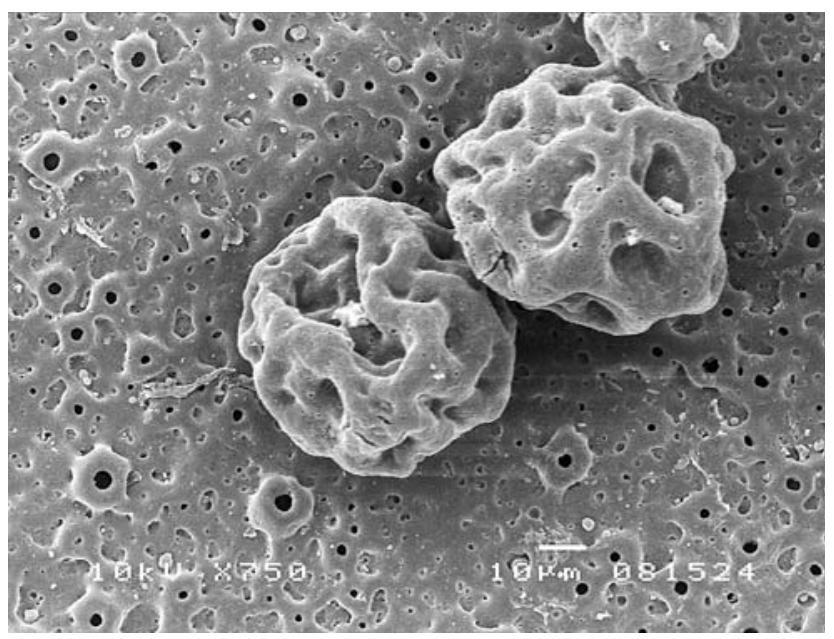

Fig. 4 Morphology of the immobilized PLGA spheres visualized by scanning electron microscopy after degradation in PBS for $800 \mathrm{~h}$ on an oxidised titanium surface $(10 \mathrm{kV}, \times 750)$

release. The microspheres were deeply eroded, showing clear diffusion paths for dexamethasone release, as well as an apparent swelling in the spheres.

\section{Discussion}

In this study a particulate drug delivery system has been incorporated into a porous oxidised titanium surface. The main aim was to assess the feasibility of such a system with respect to a sustained drug release. It has been shown before [27] that PLGA spheres of this type show a triphasic release profile, with a burst, a lag and a linear phase.

The release profiles in Fig. 3 show a similar general trend between the free system and both thermally attached systems when incubated at $37^{\circ} \mathrm{C}$ in PBS, however a few changes could be noticed upon closer inspection. The first one is the increase in burst release for the attached systems. The drug release before $100 \mathrm{~h}$ is considered the burst phase [28]. It is associated with the surface release of drugs, freed initially by the slow penetration of water into the structure of the polymer from solution. PLGA reveals a very strong increase of degradation rate as the incubation temperature increases [5]. An increase in incubation temperature from 37 to $47^{\circ} \mathrm{C}$ over a period of more than 17 days more than doubles the rate of polymer degradation. Consequently, one could consider that during the thermal attachment process $\left(1 \mathrm{~h}\right.$ at $\left.50^{\circ} \mathrm{C}\right)$ the residual water from deposition could already begin hydrolysing the polymer, potentially inducing the observed increase in burst release magnitude when compared to the free system.

Between approximately 100 and $300 \mathrm{~h}$, the drug release is almost zero order and can be considered to be in a lag phase. This period is associated with the continuing inclusion of water into the polymer structure, but while the average diffusion path length to solution of a drug molecule is increasing [28]. At this point, the polymer has yet to break down and result in the later drug release. All three release profiles show this phase, a clear release rate reduction after $100 \mathrm{~h}$.

After $300 \mathrm{~h}$ the release profile changed to a first order, linear phase, associated with the breakdown of the polymer and release of bioactives from the inner core [28]. SEM investigation evidenced severe sphere breakdown after $800 \mathrm{~h}$ (Fig. 4). The attached microsphere systems show a lower drug release rate during this phase compared to the free system, although this is not statistically different. In these cases it is important to note that the sphere size distribution does not change, thus this effect comes most probably from the effects of the attachment process, either from the thermal treatment, resultant agglomeration, or the surface itself. Previous experiments with PLGA microspheres have shown that by immobilising spheres onto the surface of a PLLA nanofibrous scaffold [29], the release profile of encapsulated drugs is significantly slowed. This is in line with other papers where PLGA spheres are immobilised in PVA [30]. Thus, immobilising PLGA microspheres on a metallic surface should also affect the release rate in the same fashion, through a barrier effect. The surface could also act to promote agglomeration and reduce the available surface area for drug release over time.

The morphological difference between oxidised (pore size $1.1 \pm 0.6 \mu \mathrm{m})$ and non-oxidised titanium is negligible when compared to the $20 \mu \mathrm{m}$ spheres. In addition, the release profile from the attached system was practically the same. Thus, it is possible to say that the porosity of the oxidised titanium surface is sufficiently different from the size of the spheres to not affect the release profile significantly.

The main aim of this investigation was assessing the feasibility of sustained drug delivery directly from a oxidised titanium disc, while maintaining the topography and cellular accessibility of the metal surface. The results shown in this paper indicate that such a surface is possible to be created with significantly longer sustained release than is typically found for films [18], and other porous surfaces on titanium [21], albeit with issues in the attachment process slightly altering the release profile of the drug delivery system used.

From the results shown in this study a few applications for this system can be envisaged, although further work is needed to reduce agglomeration, enhance sphere attachment, and control the release kinetics. Previous work on dexamethasone eluting systems for immunosuppression [26] used $0.5 \mathrm{mg}$ of $20 \mu \mathrm{m}$ loaded spheres to successfully reduce inflammation around a $1 \mathrm{~cm}$ long cotton suture. In the present study, approximately $10 \mathrm{mg}$ of microspheres 
were used, i.e. 20 times more dexamethasone, although on an implant significantly larger in size. Thus, it can be considered that this level of loading might be sufficient for a prolonged anti-inflammatory release system from the surface of a titanium implant. This system can also be considered for the improvement of bone cell adhesion (through porosity) as well as for cellular differentiation in an orthopedic implant as dexamethasone can act as a differentiation agent [24, 31].

By using another drug loaded into the PLGA spheres, it is relatively easy to extend the application range of the system investigated. Cellular proliferation in tissue scaffolds has been researched extensively [29], and such a delivery system could be applied directly onto the titanium surface, using a morphogenetic protein or a growth factor.

\section{Conclusions}

In this study a particulate drug delivery system was loaded on a oxidised titanium surface with the aim to achieve a sustained drug release whilst preserving the underlying morphology. Research into the morphology and function of this dexamethasone eluting drug delivery system showed that thermal attachment did not alter the sphere shape and size dramatically and only had a noticeable effect on drug release before $100 \mathrm{~h}$. Thus, this system allows the retention of the porous metallic topography and maintains a sustained release of dexamethasone from the attached $20 \mu \mathrm{m}$ spheres for the period of 41 days.

Acknowledgments The support of Kees Kwakernaak from the Department of Materials Science and Engineering and Michel van der Brink from the Laboratory for Process Equipment for the assistance with scanning electron microscopy is appreciated greatly.

Open Access This article is distributed under the terms of the Creative Commons Attribution Noncommercial License which permits any noncommercial use, distribution, and reproduction in any medium, provided the original author(s) and source are credited.

\section{References}

1. British National Joint Registry. NJR StatsOnline In: British national joint registry. 2008. http://www.njrcentre.org.uk/. Accessed 15 Nov 2008.

2. Smith LJ, Swaim JS, Yao C, Haberstroh KM, Nauman EA, et al. Increased osteoblast cell density on nanostructured PLGA-coated nanostructured titanium for orthopedic applications. Int J Nanomedicine. 2007;2:493-9.

3. Wise DL, Rosenkrantz H, Gregory JB, Esber HJ. Long-term controlled delivery of levonorgestrel in rats by means of small biodegradable cylinders. J Pharm Pharmacol. 1980;32:399-403.
4. Wu XS, Wang N. Synthesis, characterization, biodegradation, and drug delivery application of biodegradable lactic/glycolic acid polymers. Part II: biodegradation. J Biomat Sci-Polym E. 2001;12:21-34.

5. Wildemann B, Sander A, Schwabe P, Lucke M, Stockle U, et al. Short term in vivo biocompatibility testing of biodegradable poly(D, L-lactide) - growth factor coating for orthopaedic implants. Biomaterials. 2005;26:4035-40.

6. Kempen DHR, Lu L, Hefferan TE, Creemers LB, Maran A, et al. Retention of in vitro and in vivo BMP-2 bioactivities in sustained delivery vehicles for bone tissue engineering. Biomaterials. 2008; 29:3245-52.

7. Deng M, Chen G, Burkley D, Zhou J, Jamiolkowski D, et al. A study on in vitro degradation behavior of a poly(glycolideco-L-lactide) monofilament. Acta Biomater. 2008;4:1382-91.

8. Anderson JM, Shive MS. Biodegradation and biocompatibility of PLA and PLGA microspheres. Adv Drug Deliver Rev. 1997; 28:5-24.

9. Li JK, Wang N, Wu XS. A novel biodegradable system based on gelatin nanoparticles and poly(lactic-co-glycolic acid) microspheres for protein and peptide drug delivery. J Pharm Sci. 1997; 86:891-5.

10. Agrawal CM, Athanasiou KA. Technique to control $\mathrm{pH}$ in vicinity of biodegrading PLA-PGA implants. J Biomed Mater Res. 1997;38:105-14.

11. Wei GB, Jin QM, Giannobile WV, Ma PX. Nano-fibrous scaffold for controlled delivery of recombinant human PDGF-BB. J Control Release. 2006;112:103-10.

12. Hickey T, Kreutzer D, Burgess DJ, Moussy F. In vivo evaluation of a dexamethasone/PLGA microsphere system designed to suppress the inflammatory tissue response to implantable medical devices. J Biomed Mater Res. 2002;61:180-7.

13. Putney SD, Burke PA. Improving protein therapeutics with sustained-release formulations. Nat Biotechnol. 1998;16:153-7.

14. Wang JJ, Chua KM, Wang CH. Stabilization and encapsulation of human immunoglobulin $\mathrm{G}$ into biodegradable microspheres. J Colloid Interf Sci. 2004;271:92-101.

15. Vega E, Gamisans F, Gracia ML, Chauvet A, Lacouloche F, Egea MA. PLGA nanospheres for the ocular delivery of flurbiprofen: drug release and interactions. J Pharm Sci. 2008;97:5306-17.

16. Niwa T, Takeuchi H, Hino T, Kawashima Y. Aerosolization of lactide glycolide copolymer (PLGA) nanospheres for pulmonary delivery of peptide-drugs. Yakugaku Zasshi. 1995;115:732-41.

17. Pan CJ, Tang JJ, Shao ZY, Wang J, Huang N. Improved blood compatibility of rapamycin-eluting stent by incorporating curcumin. Colloid Surf B. 2007;59:105-11.

18. Pan CJ, Tang JJ, Weng YJ, Wang J, Huang N. Preparation and characterization of rapamycin-loaded PLGA coating stent. J Mater Sci-Mater M. 2007;18:2193-8.

19. Navarro M, Michiardi A, Castano O, Planell JA. Biomaterials in orthopaedics. Journal of the Royal Society Interface. 2008;5: 1137-58.

20. Boyan BD, Lohmann CH, Dean DD, Sylvia VL, Cochran DL, et al. Mechanisms involved in osteoblast response to implant surface morphology. Ann Rev Mat Res. 2001;31:357-71.

21. Aves EP, Estevez GF, Sader MS, Sierra JCG, Yurell JCL, et al. Hydroxyapatite coating by sol-gel on Ti-6Al-4 V alloy as drug carrier. J Mater Sci-Mater M. 2009;20:543-7.

22. Xiao JW, Zhu YC, Liu YY, Zeng Y, Xu FF. An asymmetric coating composed of gelatin and hydroxyapatite for the delivery of water insoluble drug. J Mater Sci-Mater M. 2009;20:889-96.

23. Zhu X, Kim K-H, Jeong Y. Anodic oxide films containing Ca and P of titanium biomaterial. Biomaterials. 2001;22:2199-206.

24. Cheng SL, Yang JW, Rifas L, Zhang SF, Avioli LV. Differentiation of human bone marrow osteogenic stromal cells in vitro: 
induction of the osteoblast phenotype by dexamethasone. Endocrinology. 1994;134:277-86.

25. Jimenez-Valero S, Santos B, Pajin F, Canton T, Lazaro E, Moreu $\mathrm{J}$, et al. Clinical outcomes of dexamethasone-eluting stent implantation in ST-elevation acute myocardial infarction. Catheter Cardio Interv. 2007;70:492-7.

26. Hickey T, Kreutzer D, Burgess DJ, Moussy F. Dexamethasone/ PLGA microspheres for continuous delivery of an anti-inflammatory drug for implantable medical devices. Biomaterials. 2002;23:1649-56.

27. Dawes GJS, Fratila-Apachitei LE, Mulia K, Apachitei I, Witkamp GJ, et al. Size effect of PLGA spheres on drug loading efficiency and release profiles. J Mater Sci-Mater M. 2009;20: 1089-94.
28. Siepmann J, Faisant N, Benoit JP. A new mathematical model quantifying drug release from bioerodible microparticles using Monte Carlo simulations. Pharmaceut Res. 2002;19:1885-93.

29. Wei GB, Jin QM, Giannobile WV, Ma PX. The enhancement of osteogenesis by nano-fibrous scaffolds incorporating rhBMP-7 nanospheres. Biomaterials. 2007;28:2087-96.

30. Liu J, Zhang SM, Chen PP, Cheng L, Zhou W, et al. Controlled release of insulin from PLGA nanoparticles embedded within PVA hydrogels. J Mater Sci-Mater M. 2007;18:2205-10.

31. Jager M, Fischer J, Dohrn W, Li XN, Ayers DC, et al. Dexamethasone modulates BMP-2 effects on mesenchymal stem cells in vitro. J Orthopaed Res. 2008;26:1440-8. 